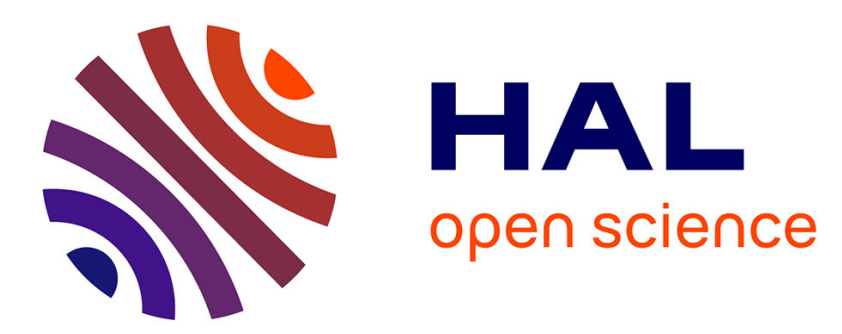

\title{
Detection of changes in the behaviour of the elderly person
}

\author{
Soumaya Msaad, Jean-Louis Dillenseger, Geoffroy Cormier, Guy Carrault
}

\section{To cite this version:}

Soumaya Msaad, Jean-Louis Dillenseger, Geoffroy Cormier, Guy Carrault. Detection of changes in the behaviour of the elderly person. 43rd Annual International Conference of the IEEE Engineering in Medicine and Biology Society (EMBC 2021), Oct 2021, Guadalajara, Mexico. 10.1109/EMBC46164.2021.9630971 . hal-03425495

\section{HAL Id: hal-03425495 \\ https://hal.science/hal-03425495}

Submitted on 10 Nov 2021

HAL is a multi-disciplinary open access archive for the deposit and dissemination of scientific research documents, whether they are published or not. The documents may come from teaching and research institutions in France or abroad, or from public or private research centers.
L'archive ouverte pluridisciplinaire HAL, est destinée au dépôt et à la diffusion de documents scientifiques de niveau recherche, publiés ou non, émanant des établissements d'enseignement et de recherche français ou étrangers, des laboratoires publics ou privés. 


\title{
Detection of changes in the behaviour of the elderly person
}

\author{
Soumaya Msaad, Jean-Louis Dillenseger, Geoffroy Cormier and Guy Carrault
}

\begin{abstract}
In this paper, we propose a solution for detecting changes in the behaviour of the elderly person based on the monitoring of activities of daily living (ADL). The elderly person's daily routine is characterized by the following five indexes: 1) percentage of time lying down, 2) percentage of time sitting, 3) percentage of time standing, 4) percentage of time absent from home, and 5) number of falls during the day. In our framework, these indexes are computed using characteristics extracted from depth and thermal data. We hypothesize that elderly persons have a well-defined, regular life routine, organized around their environment, habits, and social relations. Then, given the indexes values, a day is defined as routine or non-routine day. Thus, looking for changes of day type allows to detect changes in a person's routine. The method has been tested on a database of depth and thermal images recorded in a nursing home over an 85 days period. These tests proved the reliability of the proposed method.

Clinical Relevance - Monitoring the daily routine provides indicators for detecting changes in the behaviour of an elderly person.
\end{abstract}

\section{INTRODUCTION}

Industrialized countries around the world are experiencing an increasing proportion of older people in their populations. Population aging is becoming one of the most important social transformations of the 21 st century, with implications for almost all sectors of society. Several research projects have been conducted to monitor the well-being of older people. The current challenge is not only to live longer, but also to live a healthier life, by preventing disability and agerelated loss of autonomy.

Projects in information and communication technologies offer new health care opportunities for older persons, such as smart homes. An intelligent home is a residence equipped with non-invasive sensors that detect the activities of the elderly person (sleep, outings, meals ...).

Different sensors have been proposed to monitor the elderly, to detect abnormal behaviour and to intervene as quickly as possible by alerting the relatives of the senior or medical professionals. Namely, falls are among the most critical anomalies that must quickly be detected and dealt with to limit potentially fatal consequences. Some works propose wearable devices that automatically detect falls and activities (sitting, lying down and standing position) using sensors such as accelerometers [1], [2]. However, such devices must be worn at all times, and the elderly person may

S. Msaad, J.-L. Dillenseger and G. Carrault are with Univ Rennes, Inserm, LTSI - UMR 1099, F-35000 Rennes, France.

soumaya.msaad@univ-rennes1.fr

jean-louis.dillensegerduniv-rennesl.fr guy. carrault@univ-rennes1.fr

G. Cormier is with NeoTec-Vision, Pace, F-35000, France.

geoffroy.cormiereneotec-vision.com forget to wear it, for example after a shower. Other sensors can be found in smart homes such as sensors placed in the ground [3], passive infrared sensors [4], acoustic sensors [5] and traditional video sensors [6] or depth [7] and thermal sensors [8]. Yet, constraints linked to the cost and intrusive nature of the sensors remain. In order to deal with cost and privacy issues, we recently developed a solution based on low-cost depth and thermal sensors [9]. In a previous work, we demonstrated the interest of this approach to detect a fall [10]. In this contribution, we propose to evaluate its interest in monitoring changes in the elderly person's routine and their frailty. In literature, it is reported that elderly person follows a well-defined routine in space, time and social relationships [11].

The second section of this paper describes the proposed method, the third section presents and discusses the results the method yielded. The last section concludes this paper and point out some further directions to be given to this work.

\section{METHOD}

The proposed method is split into 4 steps as shown on Fig. 1.

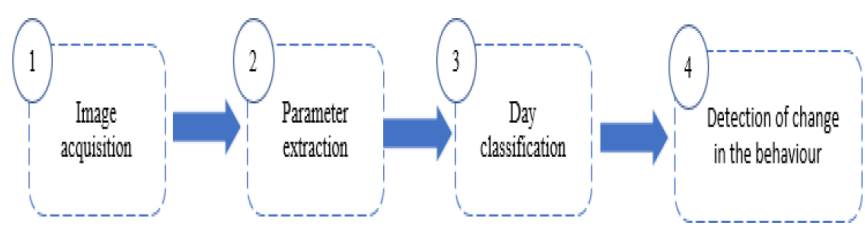

Fig. 1. Behaviour change detection framework

A device comprising depth and thermal sensors (step 1) is placed in a room (for our experiment, it was a room in a nursing home for a dependent elderly person). This device consisted in calibrated, paired depth and thermal sensors as described in [9], where the depth sensor was a Kinect-like sensor $(640 \times 480$ px @ $30 \mathrm{fps})$, and the thermal sensor was a FLIR Lepton-like thermal camera $(80 \times 60$ px @ 9 fps $)$.

Depth and thermal images, recorded every 15 minutes, provide information on the person's presence, activity (lying down or sitting or standing) and potential falls. From the images (step 2), parameters are extracted that allow the computation of behaviour indexes: the percentage of time in the lying down position (\% TLP), in a sitting position (\% TSP), in standing position (\% TStP), the percentage absent during the day (\%TAD) and the number of falls (NF). From this set of routine indexes, the type of the day is classified with machine learning as a routine day or non-routine day (step 3). In this work, three machine learning methods were 
compared: Binary Regression Logistic, Decision Tree, and Random Forest. Then, we follow the maintenance of the person's routine over time, and change of behaviour of the person can be detected (step 4).

\section{A. Posture and fall detection}

The images in Fig. 2 illustrate some examples of recorded depth images of a person.

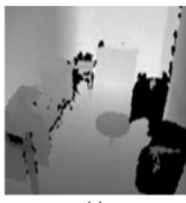

(a)

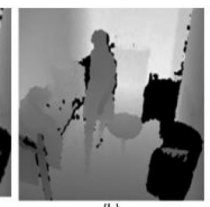

(b)

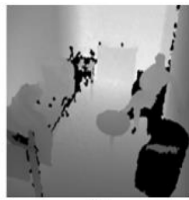

(c)

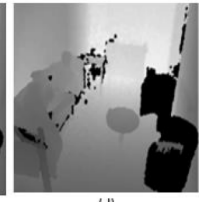

(d)
Fig. 2. Depth images. a: The person is absent. b: The person is standing c: The person is sitting. d: The person is lying down on the bed.

The images in Fig. 3 illustrate some examples of recorded thermal images of a person.

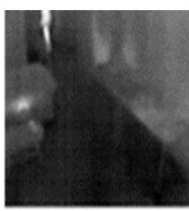

(a)

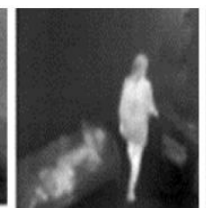

(b)

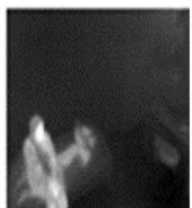

(c)

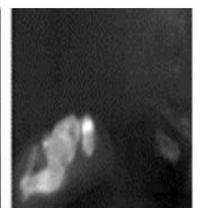

(d)
Fig. 3. Thermal images. a: The person is absent. b: The person is standing. c: The person is sitting. $\mathrm{d}$ : The person is lying down on the bed.

In previous work [10], we were able to show that depth image processing allows us to estimate the following parameters: the 3D point $H$ corresponding to the head, the height Hhead of the head with respect to the floor, and the visible proportion Near of the individual which lies under a predefined height $p_{h}$. These parameters can be used to determine the position of the person: lying down, sitting, standing, or if the person is absent.

The lying down position is determined when the visible proportion Near of the individual which lies under a predefined height $p_{h}$ is close to zero. Otherwise, the person is sitting or standing. We differentiate the "sitting" position from the "standing" position by comparing Hhead with the height $h_{H}$ when the person is standing:

1) if Hhead $\in\left[h_{H}-\delta, h_{H}+\delta\right]$ then the person is standing.

2) if Hhead $<h_{H}-\delta$ then the person is sitting.

The duration of each posture during the day gives us the percentage time index of the sitting position, of the lying down position and of the standing position. The presence or absence of the person is detected from the thermal images (the absence of hot spots in the thermal image indicates that the person is absent).

Concerning falls, we proposed in previous work [10] a method of fall detection based on the classification of the parameters extracted from the depth images. We obtained good performance with $99 \%$ of accuracy.

The measurements of posture (sitting, lying down, and standing), absence of the person, and number of falls are used to calculate indices such as \%TLP, \% TSP, \% TStP, \% TAD and NF. These indicators of behaviour change are used to reflect the defined routine of an elderly person as reported in [11].

\section{B. Methods for routine day classification}

The previous indexes characterize the person's day. A day is considered normal if it describes the routine of the person, and non-routine if it does not. The classification of the type of day in daytime daily routine or non-routine daily day can be seen as a classification/prediction problem based on the parameters that characterize this day (see below for further details). It is for this reason that, as a first step, it appeared interesting to classify the type of the day with machine learning approach. Three machine learning approaches were compared: Binary Regression Logistic (BRL), Decision Tree (DT), and Random Forest (RF). We consider BRL because the classification problem is reduced to a binary classification problem and it is a standard approach. The DT was retained because it provides interpretable decision rules. RF is recognized as robust while it performs training on several decision trees from different subsets of data. The performance measures were classically evaluated by computing the accuracy, specificity, and sensitivity of each method [12].

\section{Behaviour change detection Index}

We hypothesize that the person follows a well-defined daily routine, covering space and time [11]). Therefore, our study defines the monitoring of the evolution of its daily routine over a long period of time as an indicator of behaviour change.

A day $n$ is noted as $D(n)=1$ if it follows the person's daily routine and $D(n)=0$ if it does not. We define a routine day when the measured indexes fall into intervals which are to be defined according to the person (see results section). The principle of behaviour change detection is based on the recursive analysis of $N$ days (see equation "(1)"). The following Behaviour Index $(B I)$ is computed:

$$
B I(n)=B I(n-1)+\frac{D(n)-D(n-P t)}{P t}
$$

$B I$ reflects the percentage of routine days over the period $P t$. It is nothing else that the recursive estimation of a mean over a period $P t$. The result of the evolution of $B I$ during the $N$-day period describes the evolution of the maintenance of the routine on a continuous basis over a sliding period Pt.

\section{RESULTS}

After a presentation of the database, we first introduce the performance detection of posture, fall, and the absence of the person. In a second step, we present the implementation of the machine learning approach to classify the type of the day. Finally, we analyse the evolution of the behaviour change detection index during our experiment. 


\section{A. The database}

The database consists in depth and thermal images recorded by depth and thermal sensors installed in an elderly person's bedroom in the Raymond Thomas retirement home in Rennes, France. The experimental procedure was approved by the ethical review board of the retirement home and person participating gave their consent and its signed agreement to take part. The images have been acquired every $15 \mathrm{~min}$. This time was estimated enough to derive a global view of the organization of the day. The database consists in 8160 depth images and 8160 thermal images recorded over 85 days, which were manually annotated. The monitored person was a dependent person with mobility problems. Their standing duration was then short and, in this case, never captured by the depth sensor even during the transition phase. The annotations are distributed as follows: 3132 image pairs correspond to the lying down position, 1555 to the sitting position, 364 to falls, and the remaining 3109 image pairs correspond to moments when the person was absent.

\section{B. Posture and fall detection performance}

The proposed method for distinguishing lying down from sitting and standing was applied. In our experience, the predefined threshold ph was set to $30 \mathrm{~cm}$ and corresponds to the height of the bed in the nursing room. The position detection method was applied on the database of an older person where each image is described by the image parameters (Hhead and Near) and by the code specifying the position (lying down or sitting). Referred to the whole database, we measured a $93 \%$ accuracy to differentiate the lying down position from the sitting. For the detection of the fall, we obtained $91 \%$ of accuracy. When the person is absent, we also observed a $100 \%$ accuracy because in the thermal images there is no hot spot.

\section{Routine day classification performance}

Fig. 4 represents the succession of the ground truth of the images sitting (blue), lying down (purple), absent (red), or falling (yellow) during the day (y-axis) and for all the days (85 days) where he was followed (x-axis).

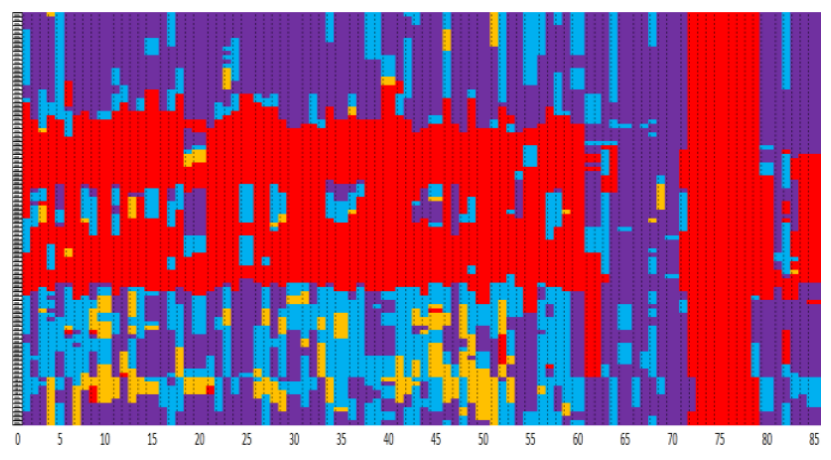

Fig. 4. Annotations of the images during 85 days. the $y$-axis shows the posture in color (see text for further details) every 15 minutes during the day and the $\mathrm{x}$-axis shows the days of recording.

We clearly see in the previous graph that the person has a routine over the first period. By viewing the images recorded from the 63rd day, we can see that the person does not leave the room for 9 days, he is often lying down on the bed. After the 9th day, the person is absent from the room for 8 days and then the person came back. This can be explained by the deterioration of the person's state of health during the 9 previous days, it justifies his absence for 8 days because the person was hospitalized. After the improvement of his condition, the person returns to his room and gradually returns progressively to his usual routine. We used this map to constitute the training set of routine and no-routine day. Indeed, we noticed that this person falls once or twice a day, and that the senior leaves the room in the morning between 7:30 am and 8:30 am to come back between 6:30 pm and 7:30 pm. At night, he sleeps between 10:30 pm and 01:30 am. The rest of the time, the person sits at the edge of the bed. This experiment leads us to measure the intervals of the indices for a routine day (TABLE I).

TABLE I

ROUTINE INTERVALS

\begin{tabular}{|c|c|}
\hline Index & routine days \\
\hline NF & {$[0,2]$} \\
\hline \%TLP & {$[14 \%, 65 \%]$} \\
\hline \% TSP & {$[4 \%, 49 \%]$} \\
\hline \% TStP & $0 \%$ \\
\hline \%TAD & {$[19 \%, 52 \%]$} \\
\hline
\end{tabular}

Binary Regression Logistic, Decision Tree, and Random Forest were used to classify the type of the day with a Leaveone-day-out cross validation method. Table II presents the performance of the three machine learning approaches for the database resulting from the detection of the posture and the fall.

TABLE II

PERFORMANCE OF THE MACHINE LEARNING ALGORITHM

\begin{tabular}{|c|c|c|c|}
\hline & Accuracy & Specificity & Sensitivity \\
\hline BLR & $74 \%$ & $87 \%$ & $52 \%$ \\
\hline DT & $88 \%$ & $91 \%$ & $84 \%$ \\
\hline RF & $92 \%$ & $93 \%$ & $90 \%$ \\
\hline
\end{tabular}

\section{Detection of behaviour change}

Fig.5 shows the evolution of the $B I$ behaviour index during 85 days over a rolling 7-day period (i.e $P t=7$ ) when using i) the ground truth annotation (blue line) and ii) the output of the classification methods (BRL, DT, RF) of the database resulting from the detection of the posture and the fall.

We observe that the three $B I$ indexes derived from BRL, DT, or RF have a trend comparable to the $B I$ index derived from the ground truth annotation. It is worthwhile to mention that RF $B I$ index confirmed the results reported in table II and is closer to the ground truth $B I$.

Briefly, we observed from day one to day 63 that $B I$ is quasi-constant $(B I \in[0.6,1])$ and translates that the routine 

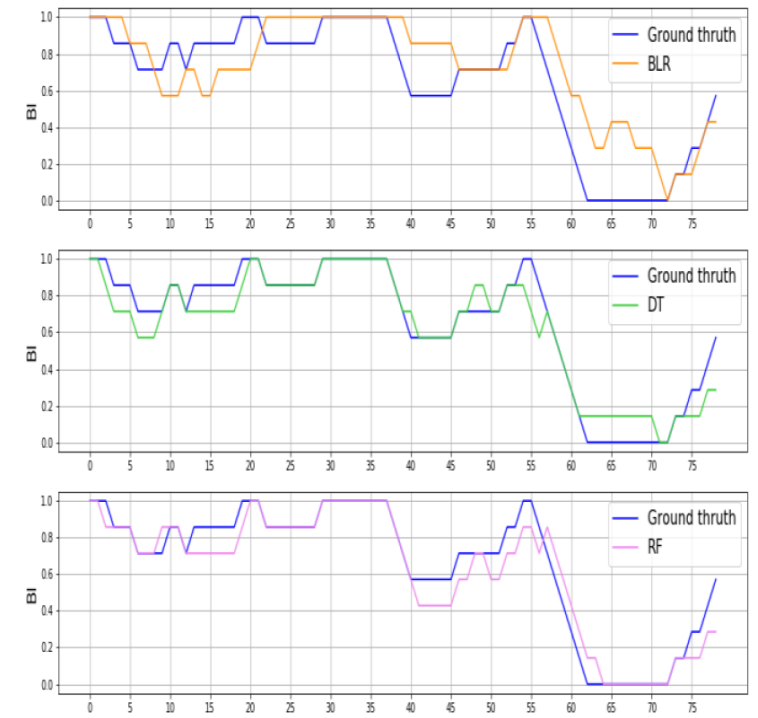

Fig. 5. Evolution of Index $B I$ derived from ground truth annotation and from the database resulting from the detection of the posture and the fall

is maintained. Then for 17 days, the person no longer follows his daily routine. Then the $B I$ increases again and a gradual return to the routine is observed. This example shows that the proposed $B I$ index highlights changes in the daily routine and could help in anticipating changes in the status of the senior.

For each day, the absolute differences between the $B I$ derived from the ground truth and from the classification approaches (BRL, DT, and RF) were computed and depicted in Fig. 6. The results confirm clearly that RF produces the minimum error.

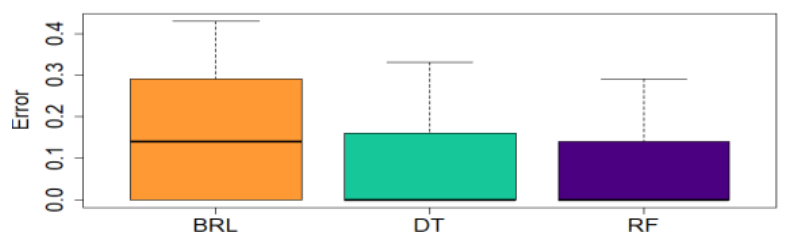

Fig. 6. Boxplot of the absolute difference between the moving average of the real data and the moving average of the predicted data.

\section{CONCLUSION}

In this article, we propose a method to detect the change in the person's behaviour based on the monitoring of the person's daily routine. It consists in measuring several indexes during the day (\%TLP, \% TSP, \% TStP, \% TAD, and NF) from depth and thermal sensors. In addition, the method is based on the classification of the day as routine or non-routine. Our result suggests that Random Forest appears as an interesting choice to perform this classification. The reliability of our method has been proven after applying the proposed method to a database of an elderly person living in a retirement home. We reached good performance: around $92 \%$ of accuracy for the classification of the type of day.

Monitoring the moving average of the days makes it possible to detect changes in the person's behaviour in a simple and easy way. Indeed, if the sliding average decreases quickly or gradually then the person's relatives or the doctor could be alerted as soon as possible. Early intervention near the person following the change in his behaviour will allow an improvement of his health in the case of deterioration, or to propose new life solutions in the case of improvement of his condition.

This work is still under development, it represents the first steps for detecting the change in the person's behaviour that will help explain and detect the change in their health status. Several perspectives can already be drawn. In spite of encouraging results in a nursing home, a first one aims at developing deep learning methods for the recognition of postures at home. In this latter case, the variety of situations will increase complexity. A second will aim to implement prediction methods (e.g. based on Kalman filters) in order to anticipate behavioural change at an early stage. We can envisage a multi-model analysis method (confrontation of a stable model and an evolving model) as already described in [13]. In the longer term, it is envisaged to design a clinical study during several months and for several seniors in order to orient progressively this work to the frailty monitoring of elderly person.

\section{ACKNOWLEDGMENT}

This work was part of the PRuDENCE project (ANR16-CE19-0015) which has been supported by the French National Research Agency (ANR). The first author received also a scholarship from the Britany Council.

\section{REFERENCES}

[1] M. Saleh and R. Le Bouquin Jeannès, "Elderly fall detection using wearable sensors: a low cost highly accurate algorithm," IEEE Sens $J$, vol. 19, no. 8, pp. 3156-3164, 2019.

[2] M. Abbas, D. Somme, and R. Le Bouquin Jeannès, "Machine learningbased physical activity tracking with a view to frailty analysis," in 42nd Ann Int Conf of the IEEE EMB (EMBC), 2020, pp. 3917-3920.

[3] Y. Zigel, D. Litvak, and I. Gannot, "A method for automatic fall detection of elderly people using floor vibrations and sound-proof of concept on human mimicking doll falls," IEEE T Bio-med Eng, vol. 56 , no. 12 , pp. $2858-2867,2009$.

[4] A. Sixsmith, N. Johnson, and R. Whatmore, "Pyroelectric IR sensor arrays for fall detection in the older population," $J$ Phys $I V$, vol. 128, pp. 153-160, 2005.

[5] Y. Li, Z.L. Zeng, M. Popescu, and K. C. Ho, "Acoustic fall detection using a circular microphone array," in 32nd Ann Int Conf of the IEEE EMB (EMBC), 2010.

[6] E. Auvinet, F. Multon, A. Saint-Arnaud, J. Rousseau, and J. Meunier, "Fall detection with multiple cameras: an occlusion-resistant method based on 3-D silhouette vertical distribution," IEEE T Inf Tech Bio, vol. 15, no. 2, pp. 290-300, 2011.

[7] E. E. Stone and M. Skubic, "Fall detection in homes of older adults using the microsoft kinect," IEEE J Biomed Health Inform, vol. 19, no. 1, pp. 290-301, 2015.

[8] L. Tao, B. Volonakis, T.and Tan, YG Jing, K. Chetty, and M. Smith, "Home activity monitoring using low resolution infrared sensor," CoRR, vol. abs/1811.05416, Nov. 2018

[9] I. Halima, J.-M. Laferté, G. Cormier, A.-J. Fougères, and J.-L. Dillenseger, "Depth and thermal information fusion for head tracking using particle filter in a fall detection context," Integrated ComputerAided Engineering,, vol. 27, no. 2, pp. 195-208, 2020.

[10] S. Msaad, G. Cormier, and G. Carrault, "Detecting falls and estimation of daily habits with depth images using machine learning algorithms," in 42nd Ann Int Conf of the IEEE EMB (EMBC), 2020. 
[11] V. Bergua, J. Bouisson, J.-F. Dartigues, J. Swendsen, C. Fabrigoule, K. Pérès, and P. Barberger-Gateau, "Restriction in instrumental activities of daily living in older persons: association with preferences for routines and psychological vulnerability," Int J Aging Hum Dev, vol. 77, no. 4, pp. 309-329, 2013.

[12] W. Zhu, N. F. Zeng, and N. Wang, "Sensitivity, specificity, accuracy, associated confidence interval and ROC analysis with practical sas implementations," in NESUG Proc., 2010, vol. 19.

[13] A. Bosnjak, G. Bevilacqua, G. Passariello, F. Mora, B. Sansó, and G. Carrault, "An approach to intelligent ischaemia monitoring," Med Biol Eng Comput, vol. 33, no. 6, pp. 749-756, 1995. 